\title{
Global Exponential Stability Criteria for Bidirectional Associative Memory Neural Networks with Time-Varying Delays
}

\author{
J. Thipcha ${ }^{1}$ and P. Niamsup ${ }^{1,2}$ \\ ${ }^{1}$ Department of Mathematics, Chiang Mai University, Chiang Mai 50200, Thailand \\ ${ }^{2}$ Centre of Excellence in Mathematics CHE, Si Ayutthaya Road, Bangkok 10400, Thailand \\ Correspondence should be addressed to P. Niamsup; piyapong.n@cmu.ac.th
}

Received 6 February 2013; Accepted 29 April 2013

Academic Editor: Yuming Chen

Copyright (C) 2013 J. Thipcha and P. Niamsup. This is an open access article distributed under the Creative Commons Attribution License, which permits unrestricted use, distribution, and reproduction in any medium, provided the original work is properly cited.

The global exponential stability for bidirectional associative memory neural networks with time-varying delays is studied. In our study, the lower and upper bounds of the activation functions are allowed to be either positive, negative, or zero. By constructing new and improved Lyapunov-Krasovskii functional and introducing free-weighting matrices, a new and improved delay-dependent exponential stability for BAM neural networks with time-varying delays is derived in the form of linear matrix inequality (LMI). Numerical examples are given to demonstrate that the derived condition is less conservative than some existing results given in the literature.

\section{Introduction}

A class of neural networks related to bidirectional associative memory (BAM) has been introduced by Kosko [1]. This model generalized the single-layer autoassociative Hebbian correlator to a two-layer pattern-matched heteroassociative circuit. It is an important model with the ability of information memory and information association, which is crucial for various applications such as pattern recognition, solving optimization problems, and automatic control engineering [2-10]. In [1, 11], Kosko investigates the global stability of BAM models and obtains a severe constraint of having a symmetric connection weight matrix. Since it is impossible to maintain an absolutely symmetric connection weight matrix, asymmetric connection has been a focus of this field. Some of these applications require that there should be a welldefined computable solution for all possible initial states. From a mathematical point of view, this means that the equilibrium point of the designed cellular neural networks (CNNs) is globally asymptotically stable (GAS) or globally exponentially stable (GES). Moreover, in biological and artificial neural networks, time delays arise in the process of information transmission; for example, in the electronic implementation of analogue neural networks, time delays occur in the communication and response of neurons owing to the finite switching speed of amplifiers. It is known that they can create an oscillatory or an unstable phenomenon. Therefore, the study of the stability and convergent dynamics of BAM neural networks with delays has raised considerable interest in recent years; see for examples [5, 7, 9, 10, 12-23] and the references cited therein. In $[14,15,18,20-22,24-$ 27], several sufficient conditions on the global exponential stability of BAM neural networks with time-varying delays have been derived. It is worth pointing out that the given criteria in $[14,15,18,20-22,24-27]$ required the following hypothesis: the time-varying delays are continuously differentiable, the derivative of time-varying delays is smaller than one, and activation functions are bounded and monotonically nondecreasing. The common approach for studying stability of BAM neural networks is Lyapunov stability theory. With a properly designed Lyapunov-Krasovskii functional as well as introducing free-weighting matrices, one may derive stability criteria in term of linear matrix inequality (LMI) which is easily solved by several available algorithms.

Based on the above discussion, we propose to study the problem of global exponential stability of BAM neural networks with time-varying delays and generalized activation functions. The main contributions of our works are 
that the system consists of both memoryless and delayed activation functions, and the lower and upper bounds of the activation functions are allowed to be either positive, negative, or zero which is more general than systems considered in $[14,15,18,21,22,24-27]$. By constructing a new and improved Lyapunov-Krasovskii functional which contains some integral terms of the activation functions, less conservative results are obtained by introducing appropriate free-weighting matrices and by using some improved integral inequality. Finally, two numerical examples are presented to show that our result is less conservative than some existing ones.

Notations. Throughout the paper, $\mathbb{R}$ denotes the set of all real numbers. $*$ denotes the elements below the main diagonal of a symmetric block matrix. diag $\{\cdots\}$ denotes the diagonal matrix. For symmetric matrices $X$ and $Y$, the notation $X>Y$ (resp., $X \geq Y$ ) means that the matrix $X-Y$ is positive definite (resp., nonnegative). $\lambda_{m}(\cdot)$ and $\lambda_{M}(\cdot)$ denote the smallest and largest eigenvalue of given square matrix, respectively.

\section{Model Description and Preliminaries}

Consider the following BAM neural network with timevarying delays of the form

$$
\begin{aligned}
\dot{u}_{i}(t)= & -c_{i} u_{i}(t)+\sum_{j=1}^{m} a_{i j}^{(1)} \widetilde{g}_{j}\left(v_{j}(t)\right) \\
& +\sum_{j=1}^{m} a_{i j}^{(2)} \widetilde{g}_{j}\left(v_{j}(t-h(t))\right)+I_{i}, \\
\dot{v}_{j}(t)= & -d_{j} v_{j}(t)+\sum_{i=1}^{n} b_{j i}^{(1)} \tilde{f}_{i}\left(u_{i}(t)\right) \\
& +\sum_{i=1}^{n} b_{j i}^{(2)} \widetilde{f}_{i}\left(u_{i}(t-d(t))\right)+I_{j}, \\
& i=1,2, \ldots, n, \quad j=1,2, \ldots, m,
\end{aligned}
$$

where $u_{i}(t)$ and $v_{j}(t)$ are the state of the $i$ th neurons from the neural field $F_{u}$ and the $j$ th neurons from the neural field $F_{v}$, at time $t$, respectively; $c_{i}$ and $d_{j}$ denote the neuron charging time constants and passive delay rates, respectively; $a_{i j}^{(1)}$ and $b_{j i}^{(1)}$ are the synaptic connection strengths; $a_{i j}^{(2)}$ and $b_{j i}^{(2)}$ are delayed synaptic connection strengths; $\widetilde{f}_{i}(\cdot)$ and $\widetilde{g}_{j}(\cdot)$ denote the activation functions of the $i$ th neurons from the neural field $F_{u}$ and the $j$ th neurons from the neural field $F_{v}$, respectively; $I_{i}$ and $I_{j}$ denote the external inputs; and $d(t)$ and $h(t)$ represent the time-varying differentiable functions which satisfy

$$
\begin{array}{ll}
\text { (i) } 0 \leq d(t) \leq d, & \dot{d}(t) \leq \tau<1, \\
\text { (ii) } 0 \leq h(t) \leq h, & \dot{h}(t) \leq \mu<1,
\end{array}
$$

where $d, h, \mu$, and $\tau$ are positive scalars.
The initial conditions associated with (1) are assumed to be

$$
\begin{array}{r}
u_{i}(s)=\widetilde{\phi}_{i}(s), \quad v_{j}(s)=\widetilde{\varphi}_{j}(s), \quad s \in[-\max \{d, h\}, 0], \\
i=1,2, \ldots, n, \quad j=1,2, \ldots, m .
\end{array}
$$

Throughout this paper, we make the following assumption on the activation function $\widetilde{f}_{i}(\cdot), \widetilde{g}_{j}(\cdot)$.

(A1) $\widetilde{f}_{i}(\cdot)$ and $\widetilde{g}_{j}(\cdot)$ are bounded on $\mathbb{R}$.

(A2) For any $\alpha, \beta \in \mathbb{R}, \alpha \neq \beta$, there exist four constant matrices $E=\operatorname{diag}\left(E_{1}, E_{2}, \ldots, E_{n}\right), F=$ $\operatorname{diag}\left(F_{1}, F_{2}, \ldots, F_{n}\right), M=\operatorname{diag}\left(M_{1}, M_{2}, \ldots, M_{m}\right)$, and $N=\operatorname{diag}\left(N_{1}, N_{2}, \ldots, N_{m}\right)$ satisfying

$$
\begin{gathered}
E_{i} \leq \frac{\tilde{f}_{i}(\alpha)-\tilde{f}_{i}(\beta)}{\alpha-\beta} \leq F_{i}, \quad i=1,2, \ldots, n, \\
N_{j} \leq \frac{\tilde{g}_{j}(\alpha)-\tilde{g}_{j}(\beta)}{\alpha-\beta} \leq M_{j}, \quad j=1,2, \ldots, m .
\end{gathered}
$$

It is clear that under (A1) and (A2), the system (1) has at least one equilibrium; see [20]. In order to simplify our proof, we shift the equilibrium point $u^{*}=\left(u_{1}^{*}, u_{2}^{*}, \ldots, u_{n}^{*}\right)^{T}, v^{*}=$ $\left(v_{1}^{*}, v_{2}^{*}, \ldots, v_{m}^{*}\right)^{T}$ of system (1) to the origin. Let $x_{i}(t)=u_{i}(t)-$ $u_{i}^{*}, y_{j}(t)=v_{j}(t)-v_{j}^{*}, f_{i}\left(x_{i}(t)\right)=\tilde{f}_{i}\left(x_{i}(t)+u_{i}^{*}\right)-\tilde{f}_{i}\left(u_{i}^{*}\right)$, $g_{j}\left(y_{j}(t)\right)=\widetilde{g}_{j}\left(y_{j}(t)+v_{j}^{*}\right)-\widetilde{g}_{j}\left(v_{j}^{*}\right), f_{i}\left(x_{i}(t-d(t))\right)=\widetilde{f}_{i}\left(x_{i}(t-\right.$ $\left.d(t))+u_{i}^{*}\right)-\widetilde{f}_{i}\left(u_{i}^{*}\right), g_{j}\left(y_{j}(t-h(t))\right)=\widetilde{g}_{j}\left(y_{j}(t-h(t))+v_{j}^{*}\right)-$ $\widetilde{g}_{j}\left(v_{j}^{*}\right), i=1,2, \ldots, n$ and $j=1,2, \ldots, m$. Then the system (1) can be transformed to

$$
\begin{aligned}
\dot{x}(t)= & -c_{i} x_{i}(t)+\sum_{j=1}^{m} a_{i j}^{(1)} g_{j}\left(y_{j}(t)\right) \\
& +\sum_{j=1}^{m} a_{i j}^{(2)} g_{j}\left(y_{j}(t-h(t))\right), \\
\dot{y}(t)= & -d_{j} y_{j}(t)+\sum_{i=1}^{n} b_{j i}^{(1)} f_{i}\left(x_{i}(t)\right) \\
& +\sum_{i=1}^{n} b_{j i}^{(2)} f_{i}\left(x_{i}(t-d(t))\right) .
\end{aligned}
$$

The activation functions $f_{i}(\cdot)$ and $g_{j}(\cdot)$ satisfy the following properties.

(H1) $f_{i}$ and $g_{j}$ are bounded on $\mathbb{R}$. 
(H2) For any $\alpha, \beta \in \mathbb{R}, \alpha \neq \beta$, there exist constant matrices $E=\operatorname{diag}\left(E_{1}, E_{2}, \ldots, E_{n}\right), F=\operatorname{diag}\left(F_{1}, F_{2}, \ldots, F_{n}\right)$, $M=\operatorname{diag}\left(M_{1}, M_{2}, \ldots, M_{m}\right)$, and $N=\operatorname{diag}\left(N_{1}\right.$, $\left.N_{2}, \ldots, N_{m}\right)$ satisfying

$$
\begin{gathered}
E_{i} \leq \frac{f_{i}(\alpha)-f_{i}(\beta)}{\alpha-\beta} \leq F_{i}, \quad i=1,2, \ldots, n, \\
N_{j} \leq \frac{g_{j}(\alpha)-g_{j}(\beta)}{\alpha-\beta} \leq M_{j}, \quad j=1,2, \ldots, m .
\end{gathered}
$$

(H3) $f_{i}(0)=0$ and $g_{j}(0)=0, i=1,2, \ldots, n, j=1,2, \ldots, m$.

Rewrite the system (7) into the vector form

$$
\begin{gathered}
\dot{x}(t)=-C x(t)+A_{1} g(y(t))+A_{2} g(y(t-h(t))), \\
\dot{y}(t)=-D y(t)+B_{1} f(x(t))+B_{2} f(x(t-d(t))) .
\end{gathered}
$$

The initial conditions associated with (7) are assumed to be

$$
\begin{array}{r}
x_{i}(s)=\phi_{i}(s), \quad y_{j}(s)=\varphi_{j}(s), \quad s \in[-\max \{d, h\}, 0], \\
i=1,2, \ldots, n, j=1,2, \ldots, m,
\end{array}
$$

where $x(t)=\left(x_{1}(t), x_{2}(t), \ldots, x_{n}(t)\right)^{T}, y(t)=\left(y_{1}(t)\right.$, $\left.y_{2}(t), \ldots, y_{m}(t)\right)^{T}, C=\operatorname{diag}\left(c_{1}, c_{2}, \ldots, c_{n}\right), \quad D=$ $\operatorname{diag}\left(d_{1}, d_{2}, \ldots, d_{n}\right), A_{1}=\left(a_{i j}^{(1)}\right)_{m \times n}, A_{2}=\left(a_{i j}^{(2)}\right)_{m \times n}, B_{1}=$ $\left(b_{i j}^{(1)}\right)_{n \times m}, B_{2}=\left(b_{i j}^{(2)}\right)_{n \times m}, f(\cdot)=\left(f_{1}(\cdot), f_{2}(\cdot), \ldots, f_{n}(\cdot)\right)^{T}$, $g(\cdot)=\left(g_{1}(\cdot), g_{2}(\cdot), \ldots, g_{m}(\cdot)\right)^{T}$.

Definition 1 (see [14]). The trivial solution of system (7) is said to be globally exponentially stable if there exist constants $k>$ 0 and $\rho \geq 1$ such that

$$
\|x(t)\|^{2}+\|y(t)\|^{2} \leq \rho e^{-2 k t}\left(\|\phi\|^{2}+\|\varphi\|^{2}\right), \quad \forall t \geq 0,
$$

where one denotes

$$
\begin{aligned}
\|\phi\|^{2}+\|\varphi\|^{2}= & \sup _{-\max \{d, h\} \leq s \leq 0}\|\phi(s)\|^{2} \\
& +\sup _{-\max \{d, h\} \leq s \leq 0}\|\varphi(s)\|^{2} .
\end{aligned}
$$

Lemma 2 (see [28]). If there exist symmetric positive-definite matrix $X_{33}>0$ and arbitrary matrices $X_{11}, X_{12}, X_{13}, X_{22}$, and $X_{23}$ such that

$$
X=\left[\begin{array}{ccc}
X_{11} & X_{12} & X_{13} \\
X_{12}^{T} & X_{22} & X_{23} \\
X_{13}^{T} & X_{23}^{T} & X_{33}
\end{array}\right] \geq 0
$$

then,

$$
\begin{aligned}
-\int_{t-h(t)}^{t} \dot{x}(s) & X_{33} \dot{x}(s) d s \\
\leq & \int_{t-h(t)}^{t}\left[\begin{array}{lll}
x^{T}(t) & x^{T}(t-h(t)) & \dot{x}^{T}(s)
\end{array}\right] \\
\times & {\left[\begin{array}{ccc}
X_{11} & X_{12} & X_{13} \\
X_{12}^{T} & X_{22} & X_{23} \\
X_{13}^{T} & X_{23}^{T} & 0
\end{array}\right]\left[\begin{array}{c}
x(t) \\
x(t-h(t)) \\
\dot{x}(s)
\end{array}\right] d s . }
\end{aligned}
$$

Lemma 3 (see [25]). For any real vectors $a, b$ and any matrix $Q>0$ with appropriate dimensions, it follows that

$$
2 a^{T} b \leq a^{T} Q a+b^{T} Q^{-1} b .
$$

Lemma 4 (see [25]). Suppose that (H2) holds; then

$$
\begin{array}{r}
\int_{v}^{u}\left[f_{i}(s)-f_{i}(v)\right] d s \leq[u-v]\left[f_{i}(u)-f_{i}(v)\right], \\
i=1,2, \ldots, n, \\
\int_{v}^{u}\left[g_{j}(s)-g_{j}(v)\right] d s \leq[u-v]\left[g_{j}(u)-g_{j}(v)\right], \\
j=1,2, \ldots, m .
\end{array}
$$

\section{Main Result}

In this section, we present a theorem which states the conditions that guarantee the global exponential stability of the system (7) employing the Lyapunov stability theory and linear matrix inequality approach.

Theorem 5. Under the assumptions (H1)-(H3), for given four diagonal matrices $E=\operatorname{diag}\left(E_{1}, E_{2}, \ldots, E_{n}\right), F=$ $\operatorname{diag}\left(F_{1}, F_{2}, \ldots, F_{n}\right), M=\operatorname{diag}\left(M_{1}, M_{2}, \ldots, M_{m}\right)$, and $N=$ $\operatorname{diag}\left(N_{1}, N_{2}, \ldots, N_{m}\right)$ and positive constants $d, h, \tau, \mu$, and $k$, the system (7) is globally exponentially stable with the convergent rate $k$, if there exist positive matrices $P_{i}, W_{i}, Z_{i}$, $i=1,2, Q_{j}, j=1,2,3,4$, positive diagonal matrices $K=$ $\operatorname{diag}\left(k_{1}, k_{2}, \ldots, k_{n}\right), R=\operatorname{diag}\left(r_{1}, r_{2}, \ldots, r_{m}\right)$, and positivedefinite matrices

$$
\begin{aligned}
& S=\left[\begin{array}{lll}
S_{11} & S_{12} & S_{13} \\
S_{12}^{T} & S_{22} & S_{23} \\
S_{13}^{T} & S_{23}^{T} & Z_{1}
\end{array}\right], \\
& T=\left[\begin{array}{lll}
T_{11} & T_{12} & T_{13} \\
T_{12}^{T} & T_{22} & T_{23} \\
T_{13}^{T} & T_{23}^{T} & Z_{2}
\end{array}\right],
\end{aligned}
$$

such that the following LMI holds:

$$
\Xi=\left[\begin{array}{cccccccc}
\Sigma_{1}^{1} & \Sigma_{2}^{1} & \Sigma_{3}^{1} & 0 & 0 & 0 & \Sigma_{7}^{1} & \Sigma_{8}^{1} \\
* & \Sigma_{2}^{2} & 0 & 0 & 0 & 0 & 0 & 0 \\
* & * & \Sigma_{3}^{3} & \Sigma_{4}^{3} & \Sigma_{5}^{3} & 0 & \Sigma_{7}^{3} & \Sigma_{8}^{3} \\
* & * & * & \Sigma_{4}^{4} & \Sigma_{5}^{4} & 0 & \Sigma_{7}^{4} & 0 \\
* & * & * & * & \Sigma_{5}^{5} & \Sigma_{6}^{5} & \Sigma_{7}^{5} & 0 \\
* & * & * & * & * & \Sigma_{6}^{6} & 0 & 0 \\
* & * & * & * & * & * & \Sigma_{7}^{7} & \Sigma_{8}^{7} \\
* & * & * & * & * & * & * & \Sigma_{8}^{8}
\end{array}\right]<0
$$


where

$$
\begin{aligned}
& \Sigma_{1}^{1}=2 k P_{1}-P_{1} C-C^{T} P_{1}-2 k E^{T} K-2 k K^{T} E \\
& +E^{T} K C+C^{T} K E+Q_{1}+d C^{T} Z_{1} C \\
& +e^{-2 k d}\left(d S_{11}+S_{13}^{T}+S_{13}\right)+W_{1}, \\
& \Sigma_{2}^{1}=e^{-2 k d}\left(d S_{12}+S_{23}^{T}-S_{13}\right), \\
& \Sigma_{3}^{1}=2 k K \text {, } \\
& \Sigma_{7}^{1}=P_{1} A_{1}-E^{T} K A_{1}-d C^{T} Z_{1} A_{1}, \\
& \Sigma_{8}^{1}=P_{2} A_{2}-E^{T} K A_{2}-d C^{T} Z_{1} A_{2} \text {, } \\
& \Sigma_{2}^{2}=e^{-2 k d}\left(d S_{22}-S_{23}^{T}-S_{23}\right)-e^{-2 k d} Q_{1}, \\
& \Sigma_{3}^{3}=h B_{1}^{T} Z_{2} B_{1}+Q_{3}-2 K C F^{-1} \text {, } \\
& \Sigma_{4}^{3}=h B_{1}^{T} Z_{2} B_{2} \text {, } \\
& \Sigma_{5}^{3}=P_{2} B_{1}-N^{T} R B_{1}-h B_{1}^{T} Z_{2} D, \\
& \Sigma_{7}^{3}=K A_{1}+R B_{1} \text {, } \\
& \Sigma_{8}^{3}=K A_{2} \text {, } \\
& \Sigma_{4}^{4}=h B_{2}^{T} Z_{2} B_{2}-(1-\tau) e^{-2 k d} Q_{3} \\
& -(1-\tau) e^{-2 k d} F^{-1} W_{1} F^{-1}, \\
& \Sigma_{5}^{4}=P_{2} B_{2}-N^{T} R B_{2}-h B_{2}^{T} Z_{2} D, \\
& \Sigma_{7}^{4}=R B_{2}, \\
& \Sigma_{5}^{5}=2 k P_{2}-P_{2} D-D^{T} P_{2}-2 k N^{T} R-2 k R^{T} N \\
& +N^{T} R D+D^{T} R N+Q_{2}+h D^{T} Z_{2} D \\
& +e^{-2 k h}\left(h T_{11}+T_{13}^{T}+T_{13}\right)+W_{2}, \\
& \Sigma_{6}^{5}=e^{-2 k h}\left(h T_{12}+T_{23}^{T}-T_{13}\right) \text {, } \\
& \Sigma_{7}^{5}=2 k R, \\
& \Sigma_{6}^{6}=e^{-2 k h}\left(h T_{22}-T_{23}^{T}-T_{23}\right)-e^{-2 k h} Q_{2} \text {, } \\
& \Sigma_{7}^{7}=d A_{1}^{T} Z_{1} A_{1}+Q_{4}-2 R D M^{-1}, \\
& \Sigma_{8}^{7}=d A_{1}^{T} Z_{1} A_{2}, \\
& \Sigma_{8}^{8}=d A_{2}^{T} Z_{1} A_{2}-(1-\mu) e^{-2 k h} Q_{4} \\
& -(1-\mu) e^{-2 k h} M^{-1} W_{2} M^{-1} \text {. }
\end{aligned}
$$

Proof. Choose the Lyapunov-Krasovskii function candidate for the system (7) to be

$$
V(t)=\sum_{l=1}^{5} V_{l}(t),
$$

where

$$
\begin{aligned}
& V_{1}(t)= e^{2 k t} x^{T}(t) P_{1} x(t) \\
&+2 \sum_{i=1}^{n} k_{i} e^{2 k t} \int_{0}^{x_{i}(t)}\left[f_{i}(s)-E_{i} s\right] d s \\
&+e^{2 k t} y^{T}(t) P_{2} y(t) \\
&+2 \sum_{j=1}^{m} r_{j} e^{2 k t} \int_{0}^{y_{j}(t)}\left[g_{j}(s)-N_{j} s\right] d s, \\
& V_{2}(t)= \int_{t-d}^{t} e^{2 k s} x^{T}(s) Q_{1} x(s) d s \\
&+\int_{t-h}^{t} e^{2 k s} y^{T}(s) Q_{2} y(s) d s, \\
&+\int_{t-h}^{t} \int_{s}^{t} e^{2 k \theta} \dot{y}^{T}(\theta) Z_{2} \dot{y}(\theta) d \theta d s . \\
& V_{3}(t)= \int_{t-d(t)}^{t} e^{2 k s} f^{T}(x(s)) Q_{3} f(x(s)) d s \\
&+\int_{t-h(t)}^{t} e^{2 k s} y^{T}(s) W_{2} y(s) d s, \\
& V_{4}(t)= \int_{t-d(t)}^{t} e^{2 k s} x^{T}(s) W_{1} x(s) d s \\
& \int_{t-d}^{t} e_{s}^{2 k \theta} \dot{x}^{T}(\theta) Z_{1} \dot{x}(\theta) d \theta d s \\
& \\
& V_{5} g(y(s)) d s,
\end{aligned}
$$

The derivative of $V(t)$ along the trajectories of system (7) is given by

$$
\begin{aligned}
\dot{V}_{1}(t)= & 2 k e^{2 k t} x^{T}(t) P_{1} x(t)+2 e^{2 k t} x^{T}(t) P_{1} \dot{x}(t) \\
& +4 k \sum_{i=1}^{n} k_{i} e^{2 k t} \int_{0}^{x_{i}(t)}\left[f_{i}(s)-E_{i} s\right] d s \\
& +2 \sum_{i=1}^{n} k_{i} e^{2 k t} \dot{x}_{i}(t)\left[f_{i}\left(x_{i}(t)\right)-E_{i} x_{i}(t)\right] \\
& +2 k e^{2 k t} y^{T}(t) P_{2} y(t)+2 e^{2 k t} y^{T}(t) P_{2} \dot{y}(t) \\
& +4 k \sum_{j=1}^{m} r_{j} e^{2 k t} \int_{0}^{y_{j}(t)}\left[g_{j}(s)-N_{j} s\right] d s \\
& +2 \sum_{j=1}^{m} r_{j} e^{2 k t} \dot{y}_{j}(t)\left[g_{j}\left(y_{j}(t)\right)-N_{j} y_{j}(t)\right]
\end{aligned}
$$




$$
\begin{aligned}
\leq e^{2 k t}\left\{2 k x^{T}(t) P_{1} x(t)+2 x^{T}(t)\right. \\
\times P_{1}\left[-C x(t)+A_{1} g(y(t))\right. \\
\left.+A_{2} g(y(t-h(t)))\right] \\
+4 k[f(x(t))-E x(t)]^{T} K x(t) \\
+2[f(x(t))-E x(t)]^{T} \\
\times K\left[-C x(t)+A_{1} g(y(t))\right. \\
\left.\quad+A_{2} g(y(t-h(t)))\right] \\
+2 k y^{T}(t) P_{2} y(t) \\
+2 y^{T}(t) P_{2}\left[-D y(t)+B_{1} f(x(t))\right. \\
\left.\quad+B_{2} f(x(t-d(t)))\right] \\
+4 k[g(y(t))-N y(t)]^{T} R y(t) \\
+2[g(y(t))-N y(t)]^{T} R \\
\times\left[-D y(t)+B_{1} f(x(t))\right. \\
\left.\left.+B_{2} f(x(t-d(t)))\right]\right\} .
\end{aligned}
$$

By (H2), we have

$$
-2 f^{T}(x(t)) K C x(t) \leq-2 f^{T}(x(t)) K C F^{-1} f(x(t)),
$$$$
-2 g^{T}(y(t)) R D y(t) \leq-2 g^{T}(y(t)) R D M^{-1} g(y(t)) \text {. }
$$

Substituting (21) into (20), we obtain

$$
\begin{aligned}
\dot{V}_{1}(t) \leq e^{2 k t} & \\
\times\{ & 2 k x^{T}(t) P_{1} x(t)-2 x^{T}(t) P_{1} C x(t) \\
& +2 x^{T}(t) P_{1} A_{1} g(y(t))+2 x^{T}(t) P_{1} A_{2} \\
& \times g(y(t-h(t)))+4 k f^{T}(x(t)) K x(t) \\
& -4 k x^{T}(t) E^{T} K x(t)-2 f^{T}(x(t)) K C \\
& \times F^{-1} f(x(t))+2 f^{T}(x(t)) K A_{1} g(y(t)) \\
& +2 f^{T}(x(t)) K A_{2} g(y(t-h(t))) \\
& +2 x^{T}(t) E^{T} K C x(t) \\
& -2 x^{T}(t) E^{T} K A_{1} g(y(t)) \\
& -2 x^{T}(t) E^{T} K A_{2} g(y(t-h(t))) \\
& +2 k y^{T}(t) P_{2} y(t)-2 y^{T}(t) P_{2} D y(t) \\
& +2 y^{T}(t) P_{2} B_{1} f(x(t))
\end{aligned}
$$$$
+2 y^{T}(t) P_{2} B_{2} f(x(t-d(t)))
$$$$
+4 k g^{T}(y(t)) R y(t)-4 k y^{T}(t) N^{T} R y(t)
$$$$
-2 g^{T}(y(t)) R D M^{-1} g(y(t))
$$$$
+2 g^{T}(y(t)) R B_{1} f(x(t))
$$$$
+2 g^{T}(y(t)) R B_{2} f(x(t-d(t)))
$$$$
+2 y^{T}(t) N^{T} R D y(t)
$$$$
-2 y^{T}(t) N^{T} R B_{1} f(x(t))
$$$$
\left.-2 y^{T}(t) N^{T} R B_{2} f(x(t-d(t)))\right\},
$$$$
\dot{V}_{2}(t)=e^{2 k t}\left\{x^{T}(t) Q_{1} x(t)\right.
$$$$
-e^{-2 k d} x^{T}(t-d) Q_{1} x(t-d)
$$$$
+y^{T}(t) Q_{2} y(t)
$$$$
\left.-e^{-2 k h} y^{T}(t-h) Q_{2} y(t-h)\right\},
$$

$\dot{V}_{3}(t)=e^{2 k t}$

$$
\times\left\{f^{T}(x(t)) Q_{3} f(x(t))\right.
$$

$-(1-\dot{d}(t)) e^{-2 k d(t)} f^{T}(x(t-d(t)))$

$\times Q_{3} f(x(t-d(t)))$

$+g^{T}(y(t)) Q_{4} g(y(t))$

$-(1-\dot{h}(t)) e^{-2 k h(t)}$

$\left.\times g^{T}(y(t-h(t))) Q_{4} g(y(t-h(t)))\right\}$

$\leq e^{2 k t}\left\{f^{T}(x(t)) Q_{3} f(x(t))\right.$

$-(1-\tau) e^{-2 k d} f^{T}(x(t-d(t)))$

$\times Q_{3} f(x(t-d(t)))$

$+g^{T}(y(t)) Q_{4} g(y(t))$

$-(1-\mu) e^{-2 k h} g^{T}(y(t-h(t)))$

$\left.\times Q_{4} g(y(t-h(t)))\right\}$,

$$
\dot{V}_{4}(t)=e^{2 k t}\left\{x^{T}(t) W_{1} x(t)\right.
$$

$-(1-\dot{d}(t)) e^{-2 k d(t)} x^{T}(t-d(t))$

$\times W_{1} x(t-d(t))$

$+y^{T}(t) W_{2} y(t)-(1-\dot{h}(t)) e^{-2 k h(t)}$

$\left.\times y^{T}(t-h(t))(s) W_{2} y^{T}(t-h(t))\right\}$ 


$$
\begin{aligned}
\leq e^{2 k t}\{ & x^{T}(t) W_{1} x(t)-(1-\tau) e^{-2 k d} \\
& \times x^{T}(t-d(t)) W_{1} x(t-d(t)) \\
& +y^{T}(t) W_{2} y(t)-(1-\mu) e^{-2 k h} \\
& \left.\times y^{T}(t-h(t))(s) W_{2} y^{T}(t-h(t))\right\} .
\end{aligned}
$$

By (H2), we have

$$
\begin{aligned}
-e^{-2 k d} & x^{T}(t-d(t)) W_{1} x(t-d(t)) \\
\leq & -e^{-2 k d} f^{T}(x(t-d(t))) F^{-1} W_{1} \\
& \times F^{-1} f(x(t-d(t))), \\
-e^{-2 k h} y^{T}(t-h(t))(s) W_{2} y^{T}(t-h(t)) & \\
\leq & -e^{-2 k h} g^{T}(y(t-h(t))) M^{-1} W_{2} \\
& \times M^{-1} g(y(t-h(t))) .
\end{aligned}
$$

By (24), we conclude that

$$
\begin{aligned}
& \dot{V}_{4}(t) \leq e^{2 k t}\left\{x^{T}(t) W_{1} x(t)-(1-\tau) e^{-2 k d}\right. \\
& \times f^{T}(x(t-d(t))) F^{-1} W_{1} F^{-1} \\
& \times f(x(t-d(t))) \\
&+y^{T}(t) W_{2} y(t)-(1-\mu) e^{-2 k h} \\
& \times g^{T}(y(t-h(t))) M^{-1} W_{2} M^{-1} \\
&\times g(y(t-h(t)))\} . \\
& \dot{V}_{5}(t)=d e^{2 k t} \dot{x}^{T}(t) Z_{1} \dot{x}(t) \\
&-\int_{t-d}^{t} e^{2 k s} \dot{x}^{T}(s) Z_{1} \dot{x}(s) d s \\
&+h e^{2 k t} \dot{y}^{T}(t) Z_{2} \dot{y}(t) \\
&-\int_{t-h}^{t} e^{2 k s} \dot{y}^{T}(s) Z_{2} \dot{y}(s) d s \\
& \leq e^{2 k t}\left\{d \dot{x}^{T}(t) Z_{1} \dot{x}(t)\right. \\
&-e^{-2 k d} \int_{t-d}^{t} \dot{x}^{T}(s) Z_{1} \dot{x}(s) d s
\end{aligned}
$$

By Lemma 2, we obtain

$$
\begin{aligned}
& -\int_{t-d}^{t} \dot{x}^{T}(s) Z_{1} \dot{x}(s) d s \\
& \leq \int_{t-d}^{t}\left[\begin{array}{lll}
x^{T}(t) & x^{T}(t-d) \dot{x}^{T}(s)
\end{array}\right] \\
& \quad \times\left[\begin{array}{lll}
S_{11} & S_{12} & S_{13} \\
S_{12}^{T} & S_{22} & S_{23} \\
S_{13}^{T} & S_{23}^{T} & 0
\end{array}\right] \\
& \quad\left[\begin{array}{c}
x(t) \\
x(t-d) \\
\dot{x}(s)
\end{array}\right] d s \\
& =\int_{t-d}^{t}\left(\begin{array}{cc}
\left.x^{T}(t) S_{11}+x^{T}(t-d) S_{12}^{T}+\dot{x}^{T}(s) S_{13}^{T}\right) x(t) d s \\
+\int_{t-d}^{t}\left(x^{T}(t) S_{12}+x^{T}(t-d) S_{22}+\dot{x}^{T}(s) S_{23}^{T}\right)
\end{array}\right. \\
& \quad \times x(t-d) d s
\end{aligned}
$$$$
+\int_{t-d}^{t}\left(x^{T}(t) S_{13}+x^{T}(t-d) S_{23}\right) \dot{x}(s) d s
$$$$
=x^{T}(t) d S_{11} x(t)+x^{T}(t-d) d S_{12}^{T} x(t)
$$$$
+\int_{t-d}^{t} \dot{x}^{T}(s) d s S_{13}^{T} x(t)
$$$$
+x^{T}(t) d S_{12} x(t-d)+x^{T}(t-d) d S_{22} x(t-d)
$$$$
+\int_{t-d}^{t} \dot{x}^{T}(s) d s S_{23}^{T} x(t-d)
$$$$
+x^{T}(t) S_{13} \int_{t-d}^{t} \dot{x}(s) d s
$$$$
+x^{T}(t-d) S_{23} \int_{t-d}^{t} \dot{x}(s) d s
$$$$
=x^{T}(t) d S_{11} x(t)+x^{T}(t-d) d S_{12}^{T} x(t)
$$$$
+x^{T}(t) d S_{12} x(t-d)
$$$$
+x^{T}(t-d) d S_{22} x(t-d)+[x(t)-x(t-d)]^{T}
$$$$
\times\left[S_{13}^{T} x(t)+S_{23}^{T} x(t-d)\right]
$$$$
+\left[x^{T}(t) S_{13}+x^{T}(t-d) S_{23}\right]
$$$$
\times[x(t)-x(t-d)],
$$ 


$$
\begin{aligned}
& -\int_{t-h}^{t} \dot{y}^{T}(s) Z_{2} \dot{y}(s) d s \\
& \leq \int_{t-h}^{t}\left[\begin{array}{llll}
y^{T}(t) & y^{T}(t-h) & \dot{y}^{T}(s)
\end{array}\right] \\
& \times\left[\begin{array}{ccc}
T_{11} & T_{12} & T_{13} \\
T_{12}^{T} & T_{22} & T_{23} \\
T_{13}^{T} & T_{23}^{T} & 0
\end{array}\right] \\
& \times\left[\begin{array}{c}
y(t) \\
y(t-h) \\
\dot{y}(s)
\end{array}\right] d s \\
& =\int_{t-h}^{t}\left(y^{T}(t) T_{11}+y^{T}(t-h) T_{12}^{T}+\dot{y}^{T}(s) T_{13}^{T}\right) y(t) d s \\
& +\int_{t-h}^{t}\left(y^{T}(t) T_{12}+y^{T}(t-h) T_{22}+\dot{y}^{T}(s) T_{23}^{T}\right) \\
& \times y(t-h) d s \\
& +\int_{t-h}^{t}\left(y^{T}(t) T_{13}+y^{T}(t-h) T_{23}\right) \dot{y}(s) d s \\
& =y^{T}(t) h T_{11} y(t)+y^{T}(t-h) h T_{12}^{T} y(t) \\
& +\int_{t-h}^{t} \dot{y}^{T}(s) d s T_{13}^{T} y(t) \\
& +y^{T}(t) h T_{12} y(t-h)+y^{T}(t-h) h T_{22} y(t-h) \\
& +\int_{t-h}^{t} \dot{y}^{T}(s) d s T_{23}^{T} y(t-h) \\
& +y^{T}(t) T_{13} \int_{t-h}^{t} \dot{y}(s) d s \\
& +y^{T}(t-h) T_{23} \int_{t-h}^{t} \dot{y}(s) d s \\
& =y^{T}(t) h T_{11} y(t)+y^{T}(t-h) h T_{12}^{T} y(t) \\
& +y^{T}(t) h T_{12} y(t-h) \\
& +y^{T}(t-h) h T_{22} y(t-h)+[y(t)-y(t-h)]^{T} \\
& \times\left[T_{13}^{T} y(t)+T_{23}^{T} y(t-h)\right] \\
& +\left[y^{T}(t) T_{13}+y^{T}(t-h) T_{23}\right] \\
& \times[y(t)-y(t-h)] .
\end{aligned}
$$

Substituting (27) into (26), we have

$$
\begin{aligned}
\dot{V}_{5}(t) \leq e^{2 k t}\{ & d \dot{x}^{T}(t) Z_{1} \dot{x}(t) \\
& +e^{-2 k d}\left[d x^{T}(t) S_{11} x(t)\right.
\end{aligned}
$$

$$
\begin{aligned}
&+ 2 d x^{T}(t) S_{12}^{T} x(t-d) \\
&+ 2[x(t)-x(t-d)]^{T} \\
& \times {\left[S_{13}^{T} x(t)+S_{23}^{T} x(t-d)\right] } \\
&+\left.d x^{T}(t-d) S_{22} x(t-d)\right] \\
&+h \dot{y}^{T}(t) Z_{2} \dot{y}(t) \\
&+e^{-2 k h}\left[h y^{T}(t) T_{11} y(t)\right. \\
&+2 h y^{T}(t) T_{12} y(t-h) \\
&+2[y(t)-y(t-h)]^{T} \\
& \times\left[T_{13}^{T} y(t)+T_{23}^{T} y(t-h)\right] \\
&\left.\left.+h y^{T}(t-h) T_{22} y(t-h)\right]\right\} .
\end{aligned}
$$

From (22), (25), and (28) we obtain

$$
\dot{V}(t) \leq e^{2 k t} \xi^{T}(t) \Xi \xi(t),
$$

where $\Xi$ is defined as in (16), and $\xi(t)=[x(t), x(t-$ $d), f(x(t)), f(x(t-d(t))), y(t), y(t-h), g(y(t)), g(y(t-h(t)))]$. Since the matrix $\Xi$ given in Theorem 5 is the negative definite matrix, we have $\dot{V}(t) \leq 0$, for all $t \geq 0$ which implies that $V(t) \leq V(0)$. From the definition of $V(t)$ in (20), we obtain

$$
\begin{aligned}
V(0)= & x^{T}(0) P_{1} x(0) \\
& +2 \sum_{i=1}^{n} k_{i} \int_{0}^{x_{i}(0)}\left[f_{i}(s)-E_{i} s\right] d s \\
& +\int_{-d}^{0} e^{2 k s} x^{T}(s) Q_{1} x(s) d s \\
& +\int_{-d(0)}^{0} e^{2 k s} f^{T}(x(s)) Q_{3} f(x(s)) d s \\
& +\int_{-d(0)}^{0} e^{2 k s} x^{T}(s) W_{1} x(s) d s \\
& +y^{T}(0) P_{2} y(0) \\
& +2 \sum_{j=1}^{m} r_{j} \int_{0}^{y_{j}(0)}\left[g_{j}(s)-N_{j} s\right] d s \\
& +\int_{-h}^{0} e^{2 k s} y^{T}(s) Q_{2} y(s) d s
\end{aligned}
$$




$$
\begin{aligned}
& +\int_{-h(0)}^{0} e^{2 k s} g^{T}(y(s)) Q_{4} g(y(s)) d s \\
& +\int_{-h(0)}^{0} y^{T}(s) W_{2} y(s) d s \\
& +\int_{-d}^{0} \int_{s}^{0} e^{2 k \theta} \dot{x}^{T}(\theta) Z_{1} \dot{x}(\theta) d \theta d s \\
& +\int_{-h}^{0} \int_{s}^{0} e^{2 k \theta} \dot{y}^{T}(\theta) Z_{2} \dot{y}(\theta) d \theta d s \\
& \leq \lambda_{M}\left(P_{1}\right)\|\phi\|^{2}+2 K_{M}(F-E)\|\phi\|^{2} \\
& +\left[\lambda_{M}\left(Q_{1}\right)+\lambda_{M}\left(Q_{3}\right)(F-E)+\lambda_{M}\left(W_{1}\right)\right] \\
& \times \int_{-d}^{0} e^{2 k s} x^{T}(s) x(s) d s+\lambda_{M}\left(P_{2}\right)\|\varphi\|^{2} \\
& +2 R_{M}(M-N)\|\varphi\|^{2} \\
& +\left[\lambda_{M}\left(Q_{2}\right)+\lambda_{M}\left(Q_{4}\right)(M-N)+\lambda_{M}\left(W_{2}\right)\right] \\
& \times \int_{-h}^{0} e^{2 k s} y^{T}(s) y(s) d s \\
& +\lambda_{M}\left(Z_{1}\right) \int_{-d}^{0} \int_{s}^{0} \dot{x}^{T}(\theta) \dot{x}(\theta) d \theta d s \\
& +\lambda_{M}\left(Z_{2}\right) \int_{-h}^{0} \int_{s}^{0} \dot{y}^{T}(\theta) \dot{y}(\theta) d \theta d s,
\end{aligned}
$$

where $K_{M}=\max _{1 \leq i \leq n}\left(k_{i}\right), F-E=\max _{1 \leq i \leq n}\left(F_{i}-E_{i}\right), R_{M}=$ $\max _{1 \leq j \leq m}\left(r_{j}\right)$ and $M-N=\max _{1 \leq j \leq n}\left(M_{j}-N_{j}\right)$.

$$
\text { It follows from Lemma } 3 \text { that }
$$

$$
\begin{aligned}
\dot{x}^{T}(\theta) \dot{x}(\theta) & \\
= & {\left[-C x(\theta)+A_{1} g(y(\theta))+A_{2} g(y(\theta-h(\theta)))\right]^{T} } \\
& \times\left[-C x(\theta)+A_{1} g(y(\theta))+A_{2} g(y(\theta-h(\theta)))\right] \\
= & x^{T}(\theta) C^{T} C x(\theta)+g^{T}(y(\theta)) A_{1}^{T} A_{1} g(y(\theta)) \\
& +g^{T}(y(\theta-h(\theta))) \times A_{2}^{T} A_{2} g(y(\theta-h(\theta))) \\
& -2 x^{T}(\theta) C^{T} A_{1} g(y(\theta))-2 x^{T}(\theta) C^{T} A_{2} \\
& \times g(y(\theta-h(\theta))) \\
& +2 g^{T}(y(\theta)) A_{1}^{T} A_{2} g(y(\theta-h(\theta)))
\end{aligned}
$$

$$
\begin{aligned}
\leq & 3 x^{T}(\theta) C^{T} C x(\theta) \\
& +3 g^{T}(y(\theta)) A_{1}^{T} A_{1} g(y(\theta)) \\
& +3 g^{T}(y(\theta-h(\theta))) A_{2}^{T} \\
& \times A_{2} g(y(\theta-h(\theta))), \\
\dot{y}^{T} & (\theta) \dot{y}(\theta) \\
= & \left.-D y(\theta)+B_{1} f(x(\theta))+B_{2} f(x(\theta-d(\theta)))\right]^{T} \\
& \times\left[-D y(\theta)+B_{1} f(x(\theta))+B_{2} f(x(\theta-d(\theta)))\right] \\
= & y^{T}(\theta) D^{T} D y(\theta)+f^{T}(x(\theta)) B_{1}^{T} B_{1} f(x(\theta)) \\
& +f^{T}(x(\theta-d(\theta))) \\
& +3 f^{T}(x(\theta-d(\theta))) B_{2}^{T} \times B_{2} g(y(\theta-h(\theta))) . \\
& +2 f^{T}(x(\theta)) B_{1}^{T} B_{2} f(x(\theta-d(\theta))) \\
& \times B_{2}^{T} B_{2} f(x(\theta-d(\theta))) \\
& -2 y^{T}(\theta) D^{T} B_{1} f(x(\theta))-2 y^{T}(\theta) D^{T} B_{2} \\
& \times f(x(\theta-d(\theta)))
\end{aligned}
$$

Substituting (31) into (30), we obtain the bound of $V(0)$ as follows:

$$
\begin{aligned}
V(0) \leq & \lambda_{M}\left(P_{1}\right)\|\phi\|^{2}+2 K_{M}(F-E)\|\phi\|^{2} \\
& +\left(\frac{1-e^{-2 k d}}{2 k}\right) \\
& \times\left[\lambda_{M}\left(Q_{1}\right)+\lambda_{M}\left(Q_{3}\right) \times(F-E)+\lambda_{M}\left(W_{1}\right)\right] \\
& +\lambda_{M}\left(P_{2}\right)\|\varphi\|^{2}+2 R_{M}(M-N)\|\varphi\|^{2} \\
& +\left(\frac{1-e^{-2 k h}}{2 k}\right) \\
& \times\left[\lambda_{M}\left(Q_{2}\right)+\lambda_{M}\left(Q_{4}\right)(M-N)+\lambda_{M}\left(W_{2}\right)\right] \\
& +\frac{d^{2}}{2} \lambda_{M}\left(Z_{1}\right)\left[3 \lambda_{M}\left(C^{T} C\right)\|\phi\|^{2}\right. \\
& +3 \lambda_{M}\left(A_{1}^{T} A_{1}\right)\|\varphi\|^{2} \\
& \left.+3 \lambda_{M}\left(A_{2}^{T} A_{2}\right)\|\varphi\|^{2}\right]
\end{aligned}
$$




$$
\begin{aligned}
+\frac{h^{2}}{2} \lambda_{M}\left(Z_{2}\right)[ & 3 \lambda_{M}\left(D^{T} D\right)\|\varphi\|^{2} \\
& +3 \lambda_{M}\left(B_{1}^{T} B_{1}\right)\|\phi\|^{2} \\
& \left.+3 \lambda_{M}\left(B_{2}^{T} B_{2}\right)\|\phi\|^{2}\right] .
\end{aligned}
$$

Thus,

$$
V(0) \leq \chi_{1}\|\phi\|^{2}+\chi_{2}\|\varphi\|^{2}
$$

where

$$
\begin{aligned}
\chi_{1}= & \lambda_{M}\left(P_{1}\right)+2 K_{M}(F-E)+\left(\frac{1-e^{-2 k d}}{2 k}\right) \\
& \times\left[\lambda_{M}\left(Q_{1}\right)+\lambda_{M}\left(Q_{3}\right)(F-E)+\lambda_{M}\left(W_{1}\right)\right] \\
& +\frac{3 d^{2}}{2} \lambda_{M}\left(Z_{1}\right) \lambda_{M}\left(C^{T} C\right) \\
& +\frac{h^{2}}{2} \lambda_{M}\left(Z_{2}\right)\left[3 \lambda_{M}\left(B_{1}^{T} B_{1}\right)+3 \lambda_{M}\left(B_{2}^{T} B_{2}\right)\right] \\
\chi_{2}= & \lambda_{M}\left(P_{2}\right)+2 R_{M}(M-N)+\left(\frac{1-e^{-2 k h}}{2 k}\right) \\
& \times\left[\lambda_{M}\left(Q_{2}\right)+\lambda_{M}\left(Q_{4}\right)(M-N)+\lambda_{M}\left(W_{2}\right)\right] \\
& +\frac{3 h^{2}}{2} \lambda_{M}\left(Z_{2}\right) \lambda_{M}\left(D^{T} D\right) \\
& +\frac{d^{2}}{2} \lambda_{M}\left(Z_{1}\right)\left[3 \lambda_{M}\left(A_{1}^{T} A_{1}\right)+3 \lambda_{M}\left(A_{2}^{T} A_{2}\right)\right]
\end{aligned}
$$

On the other hand, we have

$$
V(t) \geq e^{2 k t}\left\{\lambda_{m}\left(P_{1}\right)\|x(t)\|^{2}+\lambda_{m}\left(P_{2}\right)\|y(t)\|^{2}\right\}
$$

Therefore,

$$
\|x(t)\|^{2}+\|y(t)\|^{2} \leq \rho e^{-2 k t}\left\{\|\phi\|^{2}+\|\varphi\|^{2}\right\}
$$

where $\rho=\max \left(\chi_{1}, \chi_{2}\right) / \min \left(\lambda_{m}\left(P_{1}\right), \lambda_{m}\left(P_{2}\right)\right) \geq 1$. Therefore, the system (7) is global exponentially stable with the convergent rate $k>0$. This completes the proof.

Remark 6. In hypothesis (H2), lower bounds $E_{i}, N_{j}$ and upper bounds $F_{i}, M_{j}, i=1,2, \ldots, n, j=1,2, \ldots, m$, of activation functions are allowed to be either positive, negative, or zero. Clearly, hypothesis (H2) in our paper is more general than those given in $[14,15,18,21,22,24-27]$. Hence, our result is less conservative than some existing results given in the literature.

\section{Numerical Examples}

Example 1. Consider the BAM neural networks in (7) with $C=\operatorname{diag}(1,1,1), D=\operatorname{diag}(2,2,2), A_{1}=B_{1}=0$,

$$
\begin{aligned}
& A_{2}=\left[\begin{array}{ccc}
0.05 & 0.25 & 0.05 \\
0.1 & 0.05 & 0.15 \\
0.15 & 0.15 & 0.05
\end{array}\right], \\
& B_{2}=\left[\begin{array}{ccc}
0.75 & 0.75 & 0.95 \\
0 & 0.50 & 0.15 \\
0.15 & 0.15 & 0.05
\end{array}\right] .
\end{aligned}
$$

In this example, the activation function and time delay are given as follows: $f_{1}(x)=f_{2}(x)=f_{3}(x)=(1 / 2)(|x+1|-\mid x-$ $1 \mid), g_{1}(y)=g_{2}(y)=g_{3}(y)=(1 / 2)(|y+1|-|y-1|), d(t)=0.5$, $h(t)=1$. It follows that $d=0.5, \tau=0.3, h=1$, and $\mu=$ 0.3 . The assumption (H2) is satisfied with $F=\operatorname{diag}(1,1,1)$, $M=\operatorname{diag}(1,1,1), E=N=0$. Let $k=0.1$. By using the LMI Toolbox in MATLAB, the LMI (16) of Theorem 5 is feasible with $k=0.1$ and a set of solutions of (16) is given by

$$
\begin{aligned}
& P_{1}=\left[\begin{array}{ccc}
28.6382 & 0.6843 & 0.7501 \\
0.6843 & 28.2061 & 1.0981 \\
0.7501 & 1.0981 & 27.7326
\end{array}\right] \text {, } \\
& P_{2}=\left[\begin{array}{ccc}
11.7631 & 0.5671 & 0.4170 \\
0.5671 & 25.8296 & 0.7934 \\
0.4170 & 0.7934 & 26.2860
\end{array}\right] \text {, } \\
& Q_{1}=\left[\begin{array}{rrr}
14.4157 & -0.0495 & -0.0610 \\
-0.0495 & 14.4217 & -0.0911 \\
-0.0610 & -0.0911 & 14.4462
\end{array}\right] \text {, } \\
& Q_{2}=\left[\begin{array}{ccc}
12.8030 & -0.9294 & -0.9704 \\
-0.9294 & 16.9982 & -0.4287 \\
-0.9704 & -0.4287 & 17.2419
\end{array}\right] \text {, } \\
& Q_{3}=\left[\begin{array}{ccc}
16.7725 & 0.4495 & 0.2528 \\
0.4495 & 15.0669 & 0.5969 \\
0.2528 & 0.5969 & 13.1708
\end{array}\right] \text {, } \\
& Q_{4}=\left[\begin{array}{rrr}
11.9146 & -0.2010 & -0.3870 \\
-0.2010 & 15.1767 & -0.6757 \\
-0.3870 & -0.6757 & 15.3739
\end{array}\right] \text {, } \\
& W_{1}=\left[\begin{array}{ccc}
14.6218 & 1.0858 & 0.9997 \\
1.0858 & 13.0260 & 1.3001 \\
0.9997 & 1.3001 & 11.6739
\end{array}\right] \text {, } \\
& W_{2}=\left[\begin{array}{ccc}
8.4065 & -0.0639 & -0.2523 \\
-0.0639 & 11.9959 & 0.1677 \\
-0.2523 & 0.1677 & 11.6957
\end{array}\right] \text {, } \\
& Z_{1}=\left[\begin{array}{ccc}
13.3993 & 0.2142 & 0.3899 \\
0.2142 & 13.5746 & 0.3941 \\
0.3899 & 0.3941 & 13.7729
\end{array}\right] \text {, } \\
& Z_{2}=\left[\begin{array}{ccc}
2.3511 & 0.7468 & 0.6736 \\
0.7468 & 11.2627 & 0.3500 \\
0.6736 & 0.3500 & 11.7724
\end{array}\right] \text {, }
\end{aligned}
$$




$$
\begin{aligned}
& S_{11}=\left[\begin{array}{rrr}
12.4617 & -0.0242 & -0.0266 \\
-0.0242 & 12.4732 & -0.0399 \\
-0.0266 & -0.0399 & 12.4949
\end{array}\right] \text {, } \\
& S_{12}=\left[\begin{array}{lll}
-1.1535 & -0.0211 & -0.0257 \\
-0.0211 & -1.1444 & -0.0335 \\
-0.0257 & -0.0335 & -1.1320
\end{array}\right] \text {, } \\
& S_{13}=\left[\begin{array}{lll}
-0.9075 & -0.0415 & -0.0544 \\
-0.0415 & -0.9093 & -0.0805 \\
-0.0544 & -0.0805 & -0.9019
\end{array}\right] \text {, } \\
& S_{22}=\left[\begin{array}{rrr}
12.2314 & -0.0115 & -0.0101 \\
-0.0115 & 12.2463 & -0.0163 \\
-0.0101 & -0.0163 & 12.2626
\end{array}\right] \text {, } \\
& S_{23}=\left[\begin{array}{lll}
1.6306 & 0.0129 & 0.0208 \\
0.0129 & 1.6430 & 0.0270 \\
0.0208 & 0.0270 & 1.6490
\end{array}\right] \text {, } \\
& T_{11}=\left[\begin{array}{ccc}
8.6800 & -0.5650 & -0.6089 \\
-0.5650 & 12.0158 & -0.3052 \\
-0.6089 & -0.3052 & 12.2653
\end{array}\right] \text {, } \\
& T_{12}=\left[\begin{array}{lll}
-1.8596 & -0.2702 & -0.2795 \\
-0.2702 & -1.2902 & -0.1294 \\
-0.2795 & -0.1294 & -1.2317
\end{array}\right] \text {, } \\
& T_{13}=\left[\begin{array}{lll}
-1.7089 & -0.3795 & -0.4069 \\
-0.3795 & -0.6642 & -0.2437 \\
-0.4069 & -0.2437 & -0.5078
\end{array}\right] \text {, } \\
& T_{22}=\left[\begin{array}{ccc}
8.2813 & -0.2103 & -0.2276 \\
-0.2103 & 10.2975 & -0.1027 \\
-0.2276 & -0.1027 & 10.4221
\end{array}\right] \text {, } \\
& T_{23}=\left[\begin{array}{lll}
1.9707 & 0.1871 & 0.1870 \\
0.1871 & 2.3062 & 0.0783 \\
0.1870 & 0.0783 & 2.3346
\end{array}\right] \text {, } \\
& K=\left[\begin{array}{ccc}
14.9427 & 0 & 0 \\
0 & 14.2734 & 0 \\
0 & 0 & 13.4467
\end{array}\right] \text {, } \\
& R=\left[\begin{array}{ccc}
5.8315 & 0 & 0 \\
0 & 8.1963 & 0 \\
0 & 0 & 8.3562
\end{array}\right] \text {. }
\end{aligned}
$$

Thus, the system (7) is 0.1 -exponentially stable and the value $\rho=13.4606$. The solution of the closed-loop system satisfies

$$
\begin{aligned}
& \|x(t)\|^{2}+\|y(t)\|^{2} \\
& \quad \leq 13.4606 e^{-2(0.1) t}\left\{\|\phi\|^{2}+\|\varphi\|^{2}\right\}, \quad \forall t \in \mathbb{R}^{+} .
\end{aligned}
$$

By applying Theorem 5 and by solving the LMI (16) using MATLAB LMI Toolbox, we obtain the convergence rate $k$ which guarantees that the global exponential stability is 0.998. In Table 1, we give comparison of maximum allowable convergence rate $k$ obtained by Theorem 5 and by other
TABLE 1: Maximum allowable convergence rate.

\begin{tabular}{lccccc}
\hline$\tau=\mu$ & 0 & 0.3 & 0.5 & 0.7 & 0.9 \\
\hline$[24]$ & - & 0.459 & 0.455 & 0.455 & 0.455 \\
{$[29]$} & - & 0.445 & 0.424 & 0.408 & 0.407 \\
{$[25]$} & - & 0.52 & 0.47 & 0.39 & 0.21 \\
This paper & 0.998 & 0.998 & 0.998 & 0.998 & 0.998 \\
\hline
\end{tabular}

methods in some previous existing results. From Table 1, it is shown that the proposed global exponential stability criterion is less conservative than those obtained in $[24,25,29]$.

Example 2. Consider the BAM neural networks in (7) with

$$
\begin{gathered}
C=\left[\begin{array}{lll}
5 & 0 & 0 \\
0 & 5 & 0 \\
0 & 0 & 5
\end{array}\right], \\
A_{1}=\left[\begin{array}{ccc}
-0.72 & -0.44 & -0.21 \\
-0.72 & -0.83 & -0.1 \\
-0.01 & 0.01 & -0.04
\end{array}\right], \\
A_{2}=\left[\begin{array}{ccc}
-0.01 & -0.12 & -0.24 \\
0.17 & -0.33 & -0.43 \\
-0.25 & 0.33 & -0.05
\end{array}\right], \\
D=\left[\begin{array}{ccc}
5 & 0 & 0 \\
0 & 5 & 0 \\
0 & 0 & 5
\end{array}\right], \\
B_{1}=\left[\begin{array}{ccc}
-0.31 & -0.31 & 0.92 \\
0.34 & -0.33 & -0.78 \\
0.34 & 0.47 & 0.25
\end{array}\right], \\
B_{2}=\left[\begin{array}{ccc}
-0.83 & -0.12 & -0.52 \\
-0.65 & 0.5 & -0.14 \\
-0.05 & -0.14 & -0.65
\end{array}\right] .
\end{gathered}
$$

In this example, the activation function and time-varying delay are given as follows: $f_{1}(x)=f_{2}(x)=f_{3}(x)=$ $(1 / 2)(|x+1|-|x-1|), g_{1}(y)=g_{2}(y)=g_{3}(y)=(1 / 2)(\mid y+$ $1|-| y-1 \mid), d(t)=\sin ^{2}(0.5 t), h(t)=0.1 \cos ^{2}(t)$; the assumption (H2) is satisfied with $E=\operatorname{diag}(-0.2,-0.25,-0.2)$, $F=\operatorname{diag}(0.3,0.4,0.5), N=\operatorname{diag}(-0.2,-0.25,-0.2), M=$ $\operatorname{diag}(0.3,0.4,0.5)$. It follows that $d=0.5, \tau=0.3, h=0.1$, and $\mu=0.3$. Let $k=0.1$. By using the LMI Toolbox in MATLAB, the LMI (16) is feasible with $k=0.1$ and a set of solutions of (16) is given by

$$
\begin{gathered}
P_{1}=\left[\begin{array}{ccc}
110.3704 & -30.1135 & -5.4152 \\
-30.1135 & 90.3013 & 8.8948 \\
-5.4152 & 8.8948 & 115.0609
\end{array}\right], \\
P_{2}=\left[\begin{array}{ccc}
43.9625 & -2.3031 & 7.3341 \\
-2.3031 & 38.1671 & 4.9969 \\
7.3341 & 4.9969 & 62.3183
\end{array}\right],
\end{gathered}
$$




$$
\begin{aligned}
& Q_{1}=\left[\begin{array}{ccc}
125.4489 & -1.8802 & 0.9399 \\
-1.8802 & 127.5495 & -1.0063 \\
0.9399 & -1.0063 & 130.0399
\end{array}\right] \text {, } \\
& Q_{2}=\left[\begin{array}{ccc}
100.3079 & -4.1254 & -0.7526 \\
-4.1254 & 101.8490 & 1.0897 \\
-0.7526 & 1.0897 & 114.9297
\end{array}\right] \text {, } \\
& Q_{3}=\left[\begin{array}{ccc}
136.0956 & -4.2919 & 38.6712 \\
-4.2919 & 90.4808 & 19.2022 \\
38.6712 & 19.2022 & 113.1213
\end{array}\right] \text {, } \\
& Q_{4}=\left[\begin{array}{ccc}
42.6986 & -34.8491 & -15.2241 \\
-34.8491 & 96.7728 & 2.0801 \\
-15.2241 & 2.0801 & 145.8684
\end{array}\right] \text {, } \\
& W_{1}=\left[\begin{array}{ccc}
140.8059 & 25.3914 & -0.9357 \\
25.3914 & 146.4301 & -4.4760 \\
-0.9357 & -4.4760 & 11.6128
\end{array}\right] \text {, } \\
& W_{2}=\left[\begin{array}{ccc}
105.4681 & -0.1405 & 5.1590 \\
-0.1405 & 107.4419 & 16.7637 \\
5.1590 & 16.7637 & 134.1991
\end{array}\right] \text {, } \\
& Z_{1}=\left[\begin{array}{ccc}
69.1695 & -24.4444 & -4.5155 \\
-24.4444 & 48.7177 & 7.8545 \\
-4.5155 & 7.8545 & 65.4657
\end{array}\right] \text {, } \\
& Z_{2}=\left[\begin{array}{ccc}
99.9490 & 1.3357 & 35.2470 \\
1.3357 & 80.2179 & 10.6682 \\
35.2470 & 10.6682 & 111.8243
\end{array}\right], \\
& S_{11}=\left[\begin{array}{ccc}
103.3199 & -2.9895 & 0.4043 \\
-2.9895 & 103.2019 & -0.1404 \\
0.4043 & -0.1404 & 106.3073
\end{array}\right] \text {, } \\
& S_{12}=\left[\begin{array}{ccc}
-2.9235 & -0.6314 & 0.1584 \\
-0.6314 & -2.4889 & -0.1672 \\
0.1584 & -0.1672 & -1.9572
\end{array}\right] \text {, } \\
& S_{13}=\left[\begin{array}{ccc}
1.0569 & -2.4649 & 0.0237 \\
-2.4649 & 0.4090 & 0.2113 \\
0.0237 & 0.2113 & 2.4210
\end{array}\right] \text {, } \\
& S_{22}=\left[\begin{array}{ccc}
97.0839 & -2.1772 & 0.0865 \\
-2.1772 & 96.3284 & 0.1980 \\
0.0865 & 0.1980 & 98.4407
\end{array}\right] \text {, } \\
& S_{23}=\left[\begin{array}{ccc}
8.9358 & -1.7429 & -0.3501 \\
-1.7429 & 7.0242 & 0.7119 \\
-0.3501 & 0.7119 & 8.3850
\end{array}\right] \text {, } \\
& T_{11}=\left[\begin{array}{ccc}
105.0332 & -0.4193 & 0.0331 \\
-0.4193 & 105.2266 & 0.3043 \\
0.0331 & 0.3043 & 107.5064
\end{array}\right] \text {, } \\
& T_{12}=\left[\begin{array}{ccc}
-1.5261 & -0.6096 & -0.1149 \\
-0.6096 & -1.2415 & 0.0894 \\
-0.1149 & 0.0894 & 0.3340
\end{array}\right] \text {, }
\end{aligned}
$$

TABLE 2: Maximum allowable convergence rate.

\begin{tabular}{lcccc}
\hline$\tau=\mu$ & 0 & 0.3 & 0.5 & 0.7 \\
Convergence rate $k$ & 0.81 & 0.638 & 0.48 & 0.221 \\
\hline
\end{tabular}

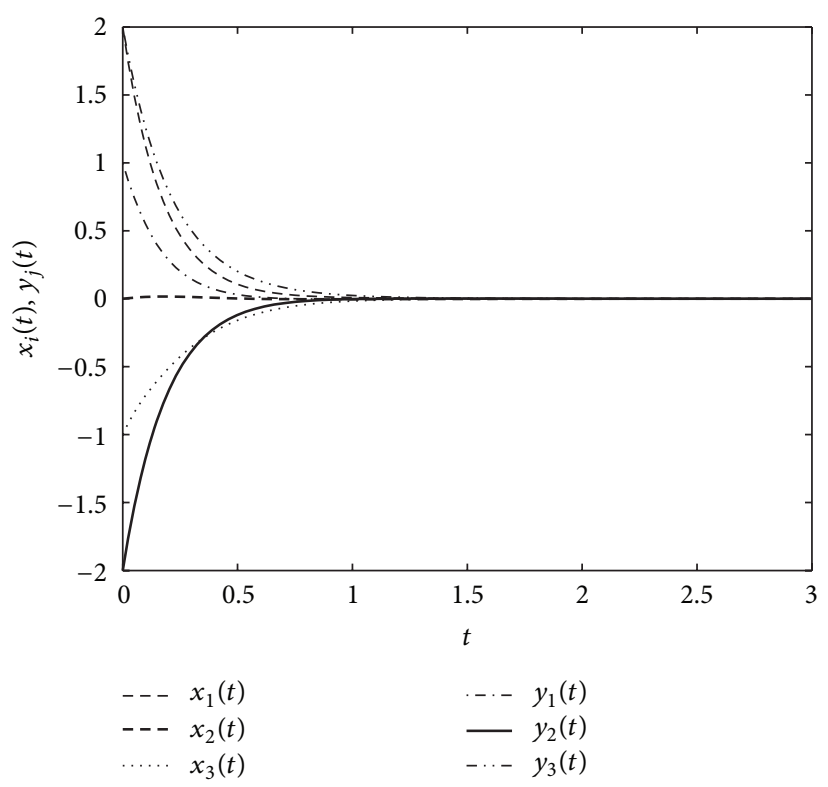

FIGURE 1: Time responses of state variables.

$$
\begin{aligned}
T_{13} & =\left[\begin{array}{ccc}
-7.6562 & -3.2414 & -0.6934 \\
-3.2414 & -5.7650 & 0.5527 \\
-0.6934 & 0.5527 & 4.8321
\end{array}\right], \\
T_{22} & =\left[\begin{array}{ccc}
104.9648 & -0.5090 & -0.0471 \\
-0.5090 & 105.1940 & 0.0964 \\
-0.0471 & 0.0964 & 106.5444
\end{array}\right], \\
T_{23} & =\left[\begin{array}{ccc}
2.6072 & 0.1420 & 0.2077 \\
0.1420 & 2.6111 & -0.0404 \\
0.2077 & -0.0404 & 2.2053
\end{array}\right], \\
K & =\left[\begin{array}{ccc}
106.5957 & 0 & 0 \\
0 & 63.3921 & 0 \\
0 & 0 & 6.3380
\end{array}\right], \\
R & =\left[\begin{array}{ccc}
86.0059 & 0 & 0 \\
0 & 72.0231 & 0 \\
0 & 0 & 71.2919
\end{array}\right]
\end{aligned}
$$

Thus, the system (7) is 0.1 -exponentially stable and the value $\rho=14.1811$. The solution of the closed-loop system satisfies

$$
\begin{aligned}
\|x(t)\|^{2} & +\|y(t)\|^{2} \\
& \leq 14.1811 e^{-2(0.1) t}\left\{\|\phi\|^{2}+\|\varphi\|^{2}\right\}, \quad \forall t \in \mathbb{R}^{+} .
\end{aligned}
$$

The maximum allowable convergence rate $k$ for different values of $\tau=\mu$ is given in Table 2 . The trajectory of solutions of BAM neural networks with time-varying delays is shown 
in Figure 1, where the initial conditions are chosen as $\phi_{1}=$ $\cos (s), \phi_{2}=\sin (s), \phi_{3}=\sin (s)-1, \varphi_{1}=\cos (s)+1, \varphi_{2}=$ $\sin (s)-2, \varphi_{3}=\cos (s)+1, s \in[-0.5,0]$.

\section{Conclusion}

This paper has proposed a new sufficient condition guaranteeing the global exponential stability criteria for bidirectional associative memory neural networks with timevarying delays and generalized activation functions. The developed stability condition is in terms of LMI, which can be easily solved by some existing software packages. Furthermore, the proposed stability conditions are less conservative than some works in the literature.

\section{Acknowledgments}

The first author is supported by the Graduate School, Chiang Mai University and the Science Achievement Scholarship of Thailand. The second author is supported by the Centre of Excellence in Mathematics, Thailand and Commission for Higher Education, Thailand.

\section{References}

[1] B. Kosko, "Adaptive bidirectional associative memories," Applied Optics, vol. 26, no. 23, pp. 4947-4987, 1987.

[2] K. Ding, N. J. Huang, and X. Xu, "Global robust exponential stability of interval BAM neural network with mixed delays under uncertainty," Neural Processing Letters, vol. 25, no. 2, pp. 127-141, 2007.

[3] C. Fu, W. Liu, and M. Yang, "Global exponential stability of a class of BAM neural networks with distributed delays," in Proceedings of the 3rd International Workshop on Advanced Computational Intelligence (IWACI '10), pp. 11-15, August 2010.

[4] M. Li, W. Chen, K. Li, X. Zhou, L. Zhao, and Y. Zhou, "Bidirectional reasoning based on BAM neural networks," in Proceedings of the 2nd International Conference on Genetic and Evolutionary Computing (WGEC '08), pp. 141-144, September 2008.

[5] X. Liao, J. Yu, and G. Chen, "Novel stability criteria for bidirectional associative memory neural networks with time delays," International Journal of Circuit Theory and Applications, vol. 30, no. 5, pp. 519-546, 2002.

[6] J. Qiu and J. Cao, "An analysis for periodic solutions of highorder BAM neural networks with delays," in Proceedings of the 2nd International Symposium on Neural Networks: Advances in Neural Networks (ISNN '05), Lecture Notes in Computer Science, pp. 288-293, Chongqing, China, June 2005.

[7] V. S. H. Rao and B. R. M. Phaneendra, "Global dynamics of bidirectional associative memory neural networks involving transmission delays and dead zones," Neural Networks, vol. 12, no. 3, pp. 455-465, 1999.

[8] Y. P. Singh, V. S. Yadav, A. Gupta, and A. Khare, "Bidirection associative memory neural networks method in the character recognition," Journal of Information Technology, Theory and Applications, pp. 382-386, 2009.
[9] Z. Zhang and K. Liu, "Existence and global exponential stability of a periodic solution to interval general bidirectional associative memory (BAM) neural networks with multiple delays on time scales," Neural Networks, vol. 24, no. 5, pp. 427-439, 2011.

[10] D. Zhou and J. Cao, "Stability conditions for bi-directional associative memory neural networks with delay," in Proceedings of the 3th World Congress on Intelligent Control and Automation, pp. 809-812, Beijing, China, July 2000.

[11] B. Kosko, "Bidirectional associative memories," IEEE Transactions on Systems, Man, and Cybernetics, vol. 18, no. 1, pp. 49-60, 1988.

[12] J. Cao and M. Dong, "Exponential stability of delayed bidirectional associative memory networks," Applied Mathematics and Computation, vol. 135, no. 1, pp. 105-112, 2003.

[13] J. Cao and L. Wang, "Exponential stability and periodic oscillatory solution in BAM networks with delays," IEEE Transactions on Neural Networks, vol. 13, no. 2, pp. 457-463, 2002.

[14] X. Huang, J. Cao, and D.-S. Huang, "LMI-based approach for delay-dependent exponential stability analysis of BAM neural networks," Chaos, Solitons \& Fractals, vol. 24, no. 3, pp. 885-898, 2005.

[15] C. Li, X. Liao, and R. Zhang, "Delay-dependent exponential stability analysis of bi-directional associative memory neural networks with time delay: an LMI approach," Chaos, Solitons \& Fractals, vol. 24, no. 4, pp. 1119-1134, 2005.

[16] X. Liao and J. Yu, "Robust stability for interval Hopfield neural networks with time delay," IEEE Transactions on Neural Networks, vol. 9, no. 5, pp. 1042-1045, 1998.

[17] X. Lou and B. Cui, "On the global robust asymptotic stability of BAM neural networks with time-varying delays," Neurocomputing, vol. 70, no. 1-3, pp. 273-279, 2006.

[18] J. H. Park, "A novel criterion for global asymptotic stability of BAM neural networks with time delays," Chaos, Solitons and Fractals, vol. 29, no. 2, pp. 446-453, 2006.

[19] A. Tian, M. Gai, B. Shi, and Q. Zhang, "Existence and exponential stability of periodic solution for a class of Cohen-Grossbergtype BAM neural networks," Neurocomputing, vol. 73, no. 16-18, pp. 3147-3159, 2010.

[20] H. Wang, Q. Song, and C. Duan, "LMI criteria on exponential stability of BAM neural networks with both time-varying delays and general activation functions," Mathematics and Computers in Simulation, vol. 81, no. 4, pp. 837-850, 2010.

[21] Y. Yuan and X. Li, "New results for global robust asymptotic stability of BAM neural networks with time-varying delays," Neurocomputing, vol. 74, no. 1-3, pp. 337-342, 2010.

[22] E. Yucel and S. Arik, "New exponential stability results for delayed neural networks with time varying delays," Physica $D$, vol. 191, no. 3-4, pp. 314-322, 2004.

[23] Z. Zhang, Y. Yang, and Y. Huang, "Global exponential stability of interval general BAM neural networks with reactiondiffusion terms and multiple time-varying delays," Neural Networks, vol. 24, no. 5, pp. 457-465, 2011.

[24] Y. Chen, S. Huang, J. Yin, and Q. Li, "Novel global exponential stability analysis for BAM neural networks with time-varying delays," in Proceedings of Chinese Control and Decision Conference (CCDC '09), pp. 4355-4360, June 2009.

[25] J. H. Park, S. M. Lee, and O. M. Kwon, “On exponential stability of bidirectional associative memory neural networks with timevarying delays," Chaos, Solitons and Fractals, vol. 39, no. 3, pp. 1083-1091, 2009. 
[26] L. Sheng and H. Yang, "Novel global robust exponential stability criterion for uncertain BAM neural networks with time-varying delays," Chaos, Solitons and Fractals, vol. 40, no. 5, pp. 2102-2113, 2009.

[27] G. Zong, Y. Wu, and L. Hou, "Global exponential stability for BAM neural networks with time-varying delays," in Proceedings of the Chinese Control and Decision Conference (CCDC '08), pp. 2500-2505, Yantai, China, July 2008.

[28] P.-L. Liu, "Robust exponential stability for uncertain timevarying delay systems with delay dependence," Journal of the Franklin Institute, vol. 346, no. 10, pp. 958-968, 2009.

[29] Y. Chen and T. Qin, "Improved global exponential stability criterion for BAM neural networks with time-varying delays," Advances in Neural Network, pp. 128-137, 2008. 


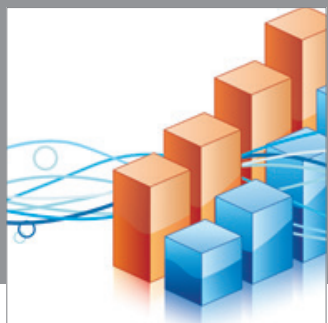

Advances in

Operations Research

mansans

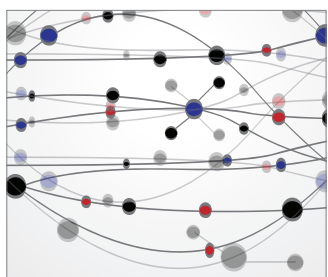

The Scientific World Journal
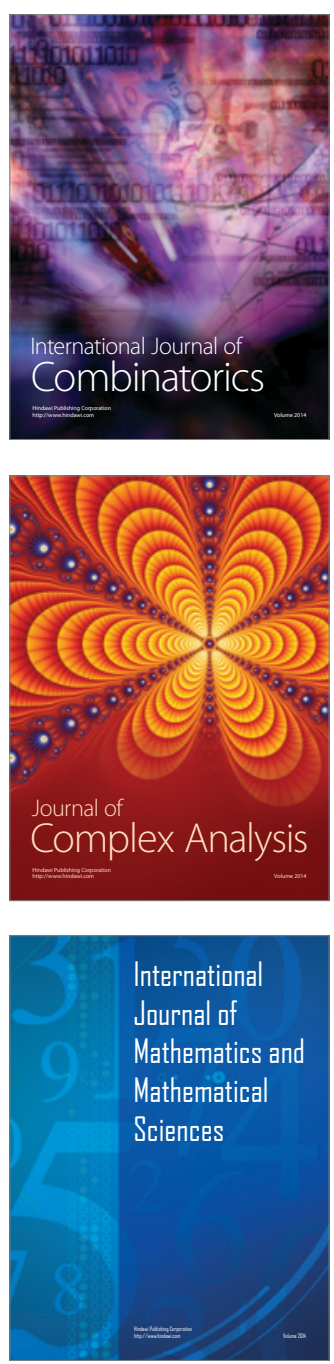
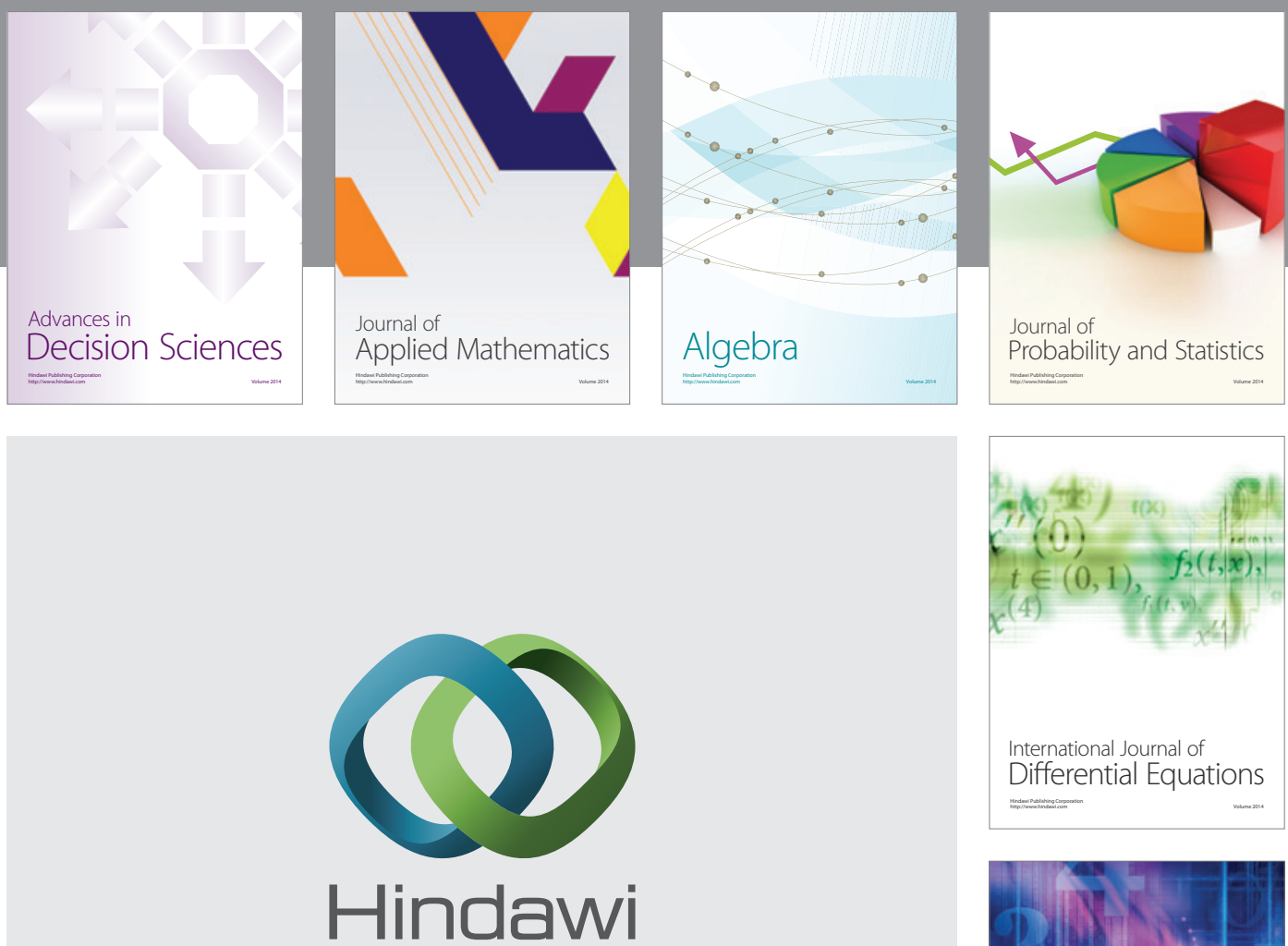

Submit your manuscripts at http://www.hindawi.com
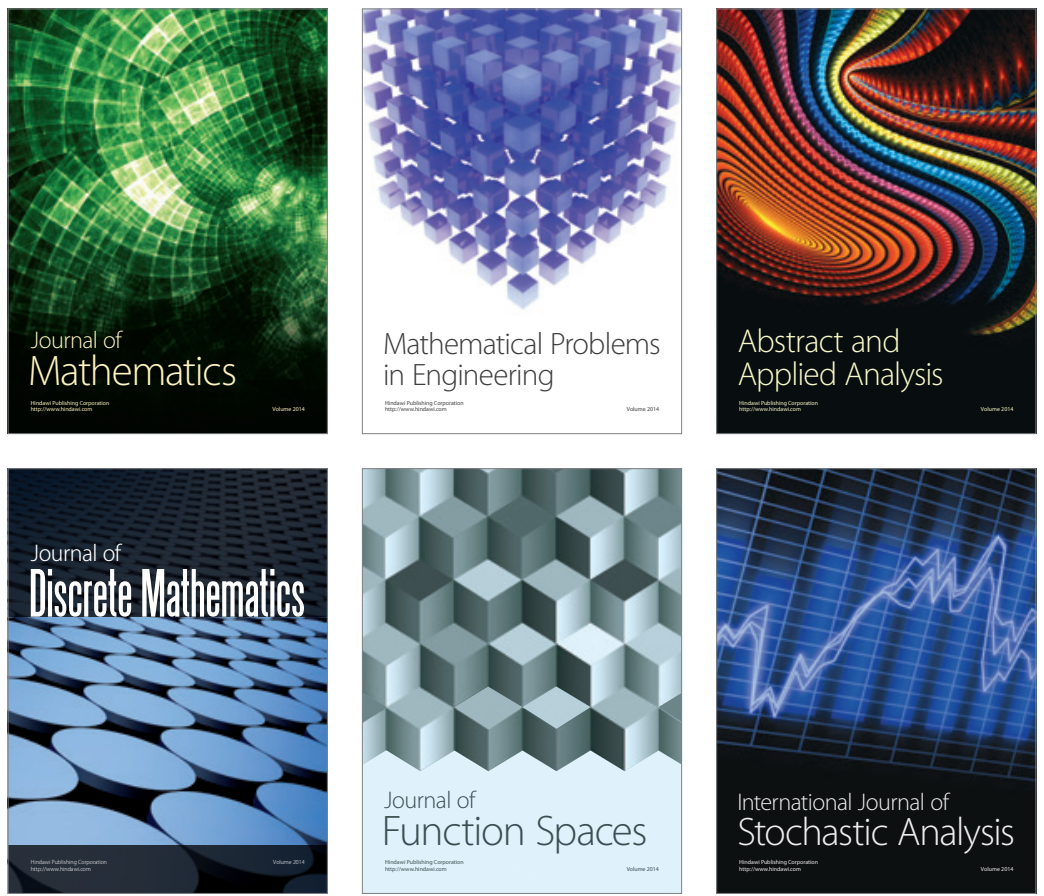

Journal of

Function Spaces

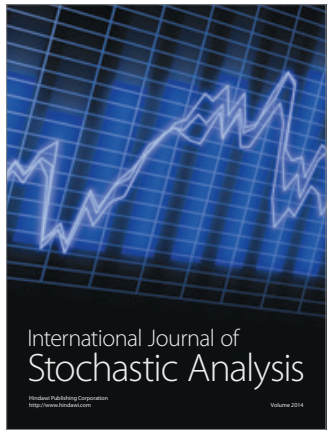

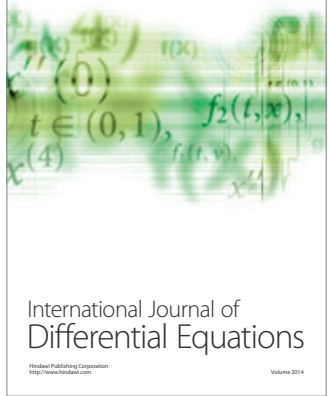
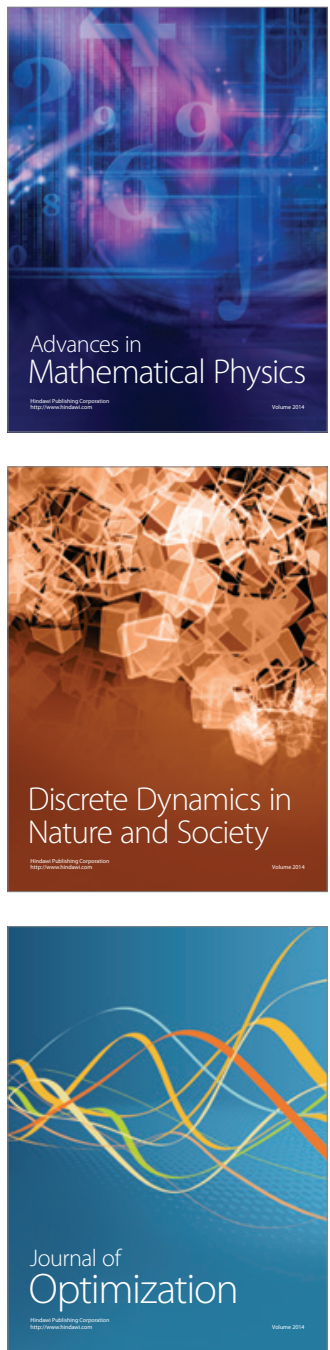